\title{
Effect of phlorizin on metabolic abnormalities in Spontaneously Diabetic Torii (SDT) rats
}

\author{
Takeshi Ohta $^{1,2}$, Hisayo Morinaga ${ }^{2}$, Takuji Yamamoto ${ }^{1,2}$, Takahisa Yamada $^{1,2^{\star}}$ \\ ${ }^{1}$ Laboratory of Animal Breeding and Genetics, Graduate School of Agriculture, Kyoto University, Kyoto, Japan \\ ${ }^{2}$ Laboratory of Animal Genetics, Graduate School of Science and Technology, Niigata University, Niigata, Japan; \\ *Corresponding Author: tyamada@agr.niigata-u.ac.jp
}

Received 12 December 2011; revised 12 January 2012; accepted 6 February 2012

\begin{abstract}
The Spontaneously Diabetic Torii (SDT) rat is a novel model for nonobese type 2 diabetes. In this study we investigated the glycolipid metabolic changes with phlorizin-treatment, which inhibits intestinal glucose uptake and renal glucose reabsorption, in male SDT rats. Phlorizin (100 mg/kg, b.i.d., s.c.) was administered for 4 weeks to SDT rats from 20 to 24 weeks of age. As a result, phlorizin reduced the development of hyperglycemia and decreased the hemoglobin A1c (HbA1c) levels. In the liver, phlorizin increased mRNA levels of glucokinase, the enzymes related with the glycogen cascade and the proteins associated with lipid metabolism. In conclusion, chronic administration of phlorizin in SDT rats produced a good glycemic control and an improvement in liver function.
\end{abstract}

Keywords: Hyperglycemia; Phlorizin; SDT Rat

\section{INTRODUCTION}

Type 2 diabetes is a polygenic disorder characterized by defects in insulin secretion and insulin sensitivity, which leads to glycolipid metabolic abnormalities [1]. Type 2 diabetes has increased worldwide in incidence with excessive calorie intake and sedentary lifestyles $[2$, $3]$. Hyperglycemia (glucotoxicity) and dyslipidemia (lipotoxicity) impair $\beta$-cell function and increase insulin resistance in the peripheral tissues, such as muscle, liver and fat tissue $[4,5]$.

The Spontaneously Diabetic Torii (SDT) rat is a model for nonobese spontaneous diabetes which was developed by Torii Pharmaceutical Co., Ltd. (Tokyo, Japan) [6,7]. Male SDT rats develop diabetes mellitus from about 20 weeks of age and diabetic complications, such as ocular lesions and nephropathy, from about 40 weeks of age [8, 9]. Moreover, SDT rats show glucose intolerance before the onset of diabetes. The glucose intolerance was investigated at 12 and 16 weeks of age [10-12]. SDT rats show a remarkable hyperglycemia after the onset of diabetes mellitus.

Phlorizin is an antidiabetic agent that is found primarily in apple peels [13]. Phlorizin inhibits intestinal glucose uptake via the sodium D-glucose cotransporter and similarly inhibits renal glucose reabsorption $[14,15]$. Correction of hyperglycemia with phlorizin, which shows a reduction of glucotoxicity, has been shown to normalize the abnormalities in the peripheral tissues and pancreas [16].

In this study, phlorizin was administered to male SDT rats, and the effects of phlorizin on the metabolic abnormalities were investigated.

\section{MATERIALS AND METHODS}

\subsection{Animals}

Male SDT rats and Sprague-Dawley (SD) rats (Clea Japan, Tokyo, Japan) were used for the study. SD rat was used as a control rat. Rats were housed in suspended bracket cages and given a standard laboratory diet (CRF1, Oriental yeast co., ltd. Tokyo, Japan) and water ad libitum in a controlled room for temperature, humidity and lightening.

\subsection{Chronic Administration of Phlorizin in SDT Rats}

Phlorizin was subcutaneously administered to SDT rats (20 weeks of age) twice a day for 4 weeks. Body weight and non-fasted serum parameters, such as glucose, insulin, triglyceride (TG), and total cholesterol (TC) levels were examined per week. Blood samples were collected from the tail vein of rats. The glucose, TG, and TC levels were measured using commercial kits (Roche Diagnostics, Basel, Switzerland) and an automatic analyzer (Hitachi, Tokyo, Japan). Serum insulin level was measured with a rat-insulin enzyme-linked immunosorbent 
assay (ELISA) kit (Morinaga Institute of Biological Science, Yokohama, Japan). At 4 weeks after phlorizin treatment, the hemoglobin A1c (HbA1c) level was measured using a commercial kit (Roche Diagnostics, Basel, Switzerland) and an automatic analyzer (Hitachi, Tokyo, Japan).

\section{3. mRNA Quantification with Real-Time Quantitative PCR}

Total RNA was extracted from the livers of rats at 24 weeks of age. RNA was transcribed into cDNA using MMLV reverse transcriptase and random primers (Invitrogen, Carlsbad, CA, USA). The reaction mixture was incubated for $10 \mathrm{~min}$ at $25^{\circ} \mathrm{C}, 1 \mathrm{~h}$ at $37^{\circ} \mathrm{C}$, and $5 \mathrm{~min}$ at $95^{\circ} \mathrm{C}$. Realtime PCR quantification was performed in a $50 \mu \mathrm{L}$ reaction mixture with an automated sequence detector combined with ABI Prism 7700 Sequence Detection System software (Applied Biosystems, Foster City, CA, USA). The reaction mixture contained $50 \mathrm{ng}$ of synthesized cDNA, $3.5 \mathrm{mM} \mathrm{MgCl}_{2}, 0.3 \mu \mathrm{M}$ primers, 0.1 $\mu \mathrm{M}$ probes, and 1.25 units of Ampli Taq Gold. Cycle parameters were $10 \mathrm{~min}$ at $95^{\circ} \mathrm{C}$, followed by 40 cycles of $15 \mathrm{~s}$ at $95^{\circ} \mathrm{C}$ and $60 \mathrm{~s}$ at $60^{\circ} \mathrm{C}$. The following primers and FAM-conjugated probes were designed using Primer Express software (Applied Biosystems, Table 1). The mRNA levels of glucokinase (GK), glucose-6-phosphatase (G-6-Pase), glycogen synthase (GS), glycogen phosphorylase (GP), sterol regulatory element-binding protein 1 (SREBP1), and microsomal triglyceride transfer protein (MTP) were quantified and normalized for the comparison against a control reaction for $18 \mathrm{~s}$ rRNA (purchased from Applied Biosystems).

\subsection{Statistical Analysis}

Results were expressed as the mean \pm standard deviation. Statistical analysis was performed using StatLight 1998(c) Yukms corp. Comparisons between two groups were made as follows: when equality of variances was indicated by an F test, statistical analysis was performed using a Student's t-test; when equality of variances was not indicated by an F test, statistical analysis was performed using an Aspin-Welch's t-test. Differences were defined as significant at $\mathrm{p}<0.05$.

\section{RESULTS AND DISCUSSION}

The chronic effects of phlorizin on the biological parameters are shown in Figure 1. Body weights in SDT rats were lower than in SD rats. Phlorizin treatment showed no change in the body weights in SDT rats (Figure 1(a)). Non-fasted serum glucose levels in SDT rats showed a remarkable increase as compared with those in SD rats (At 24 weeks of age, SDT rats, $716.8 \pm 34.8$ $\mathrm{mg} / \mathrm{dl}$ vs. SD rats, $131.0 \pm 4.6 \mathrm{mg} / \mathrm{dl})$. Phlorizin treatment improved the hyperglycemia from 21 to 24 weeks of age in SDT rats (Figure 1(b)) and showed a significant reduction in the HbA1c level at 24 weeks of age (Control, 10.2\% $\pm 2.2 \%$ vs. Phlorizin treatment, $6.6 \% \pm$ $1.2 \%$ ) (Figure 2). Chronic treatment with phlorizin induced good glycemic control in SDT rats. Non-fasted serum insulin levels in SDT rats were lower than in SD rats and the insulin levels in SDT rats with phlorizin treatment did not show significant changes (Figure 1(c)). Non-fasted serum TG levels in SDT rats increased only at 24 weeks of age as compared with those in SD rats. The TG levels in SDT rats with phlorizin decreased from 23 to 24 weeks of age (Figure 1(d)). Non-fasted serum TC levels in SDT rats were lower than those in SD rats with a significant change at 23 weeks of age. Phlorizin treatment showed no changes in the TC levels in SDT rats (Figure 1(e)).

Phlorizin improved the hyperglycemia in other diabetic models. In Goto-Kakizaki rats, which is a nonobese animal model of type 2 diabetes, phlorizin decreased the blood glucose levels and improved the glucose stimulated insulin secretion [17]. In BioBreeding (BB) rats, which is an animal model for type 1 diabetes, phlorizin with 5-aminoimidazole-4-carboxyamide ribonucleoside (AICAR) decreased the blood glucose levels and reversed hypoglycemia-specific defects in glucagon secretion [18]. It is considered that phlorizin induces an improvement of glycometabolic abnormalities by ameliorating glucotoxicity.

In addition to analyses of the biological parameters, mRNA expression of the enzymes and proteinsrelated to glycolipid metabolism in the liver were measured to examine how phlorizin treatment in SDT rats affects the function of the glycolipid metabolism. mRNA expression

Table 1. Primers and probes for real time PCR reactions.

\begin{tabular}{clll}
\hline Gene & \multicolumn{1}{c}{ Forward primers (sense) } & \multicolumn{1}{c}{ Reverse primers (antisense) } & \multicolumn{1}{c}{ Probes } \\
\hline GK & AGCAGATCCACAACATCCTAAGC & TCCTGCGGAGCACATATGG & CGACCCTCTGTCACCGACTGC \\
G-6-Pase & GAAGGCCAAGAGATGGTGTGA & TGCAGCTCTTGCGGTACATG & CCACCTTGACACTACACCCTTTGCCAG \\
GS & AGAAATCACAGCCATCGAGGC & GTTCAAGCCGTTTGGAGTCAC & ACGACATGCTGAAGAGGAAGCCTGATGT \\
GP & GAGCACCCAATGACTTTAACCTTC & TATTCTCAGCCAGGTTCCGGT & TGTCGGAGACTACATTCAGGCTGTGCTG \\
SREBP1 & CGACTACATCCGCTTCTTACAGC & TTTTGTGAGCACTTCGCAGG & CAGCAACCAGAAACTCAAGCAGGAGAACC \\
MTP & GGACGTTGTGTTACTGTGGAGG & ACATTGACAGCCGTTATCGTGA & GATCCCGACGGTGACGATGATCAACTG \\
\hline
\end{tabular}




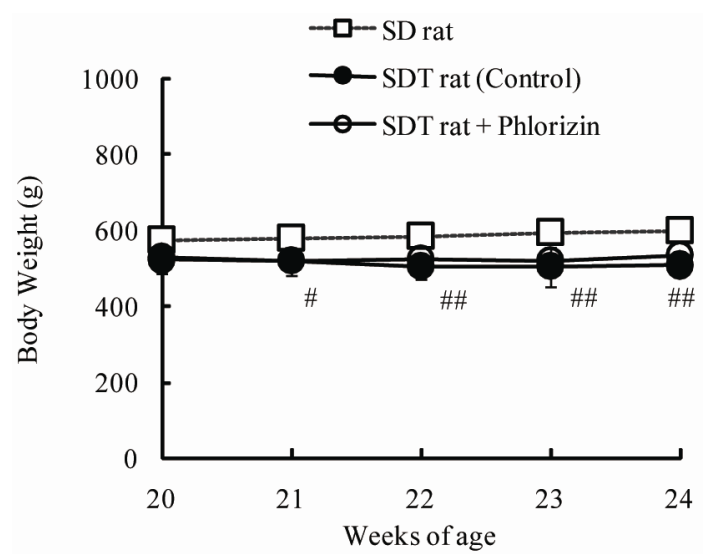

(a)

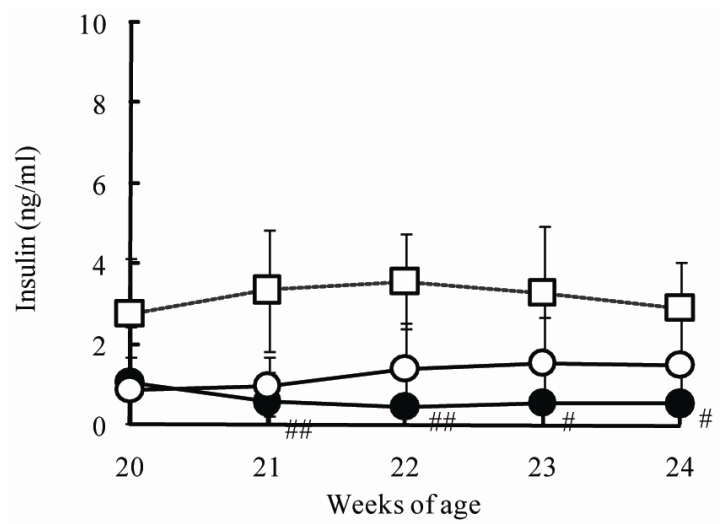

(c)

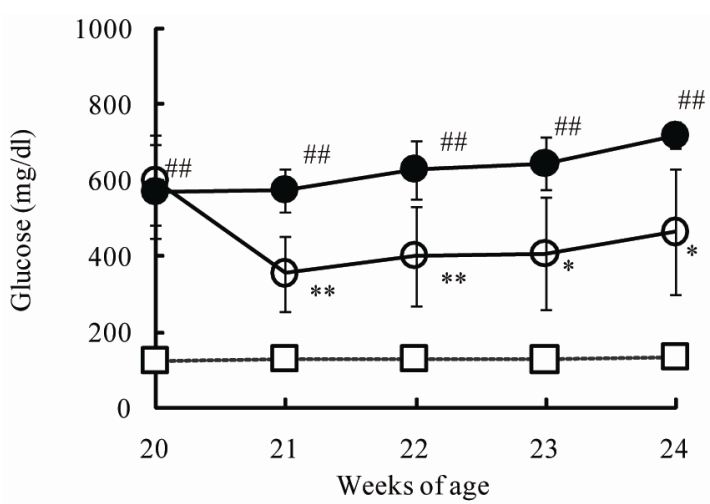

(b)

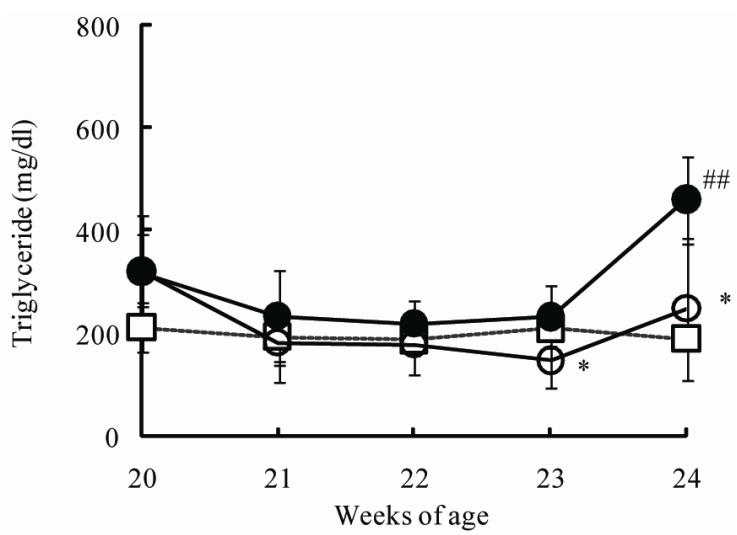

(d)

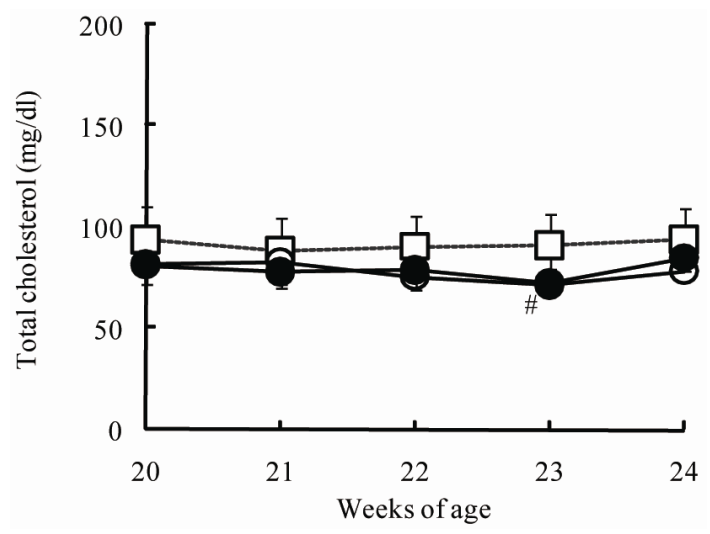

(e)

Figure 1. Effect of phlorizin on body weight (a), serum glucose (b), insulin (c), TG (d), and TC (e) levels in SDT rats. Phlorizin was administered for 4 weeks, from 20 to 24 weeks of age. Data represent mean \pm standard deviation $(n=5-$ 6). ${ }^{\#} \mathrm{p}<0.05,{ }^{\# \#} \mathrm{p}<0.01$; significantly different from SD rat. ${ }^{*} \mathrm{p}<0.05,{ }^{* *} \mathrm{p}<0.01$; significantly different from SDT rat (Control).

of GK, which is a limited enzyme in the glycolysis pathway, in SDT rats decreased as compared with that in SD rats (Table 2). The mRNA expression showed a significant increase by phlorizin treatment. On the other hand, mRNA expression of G6Pase, which controls hepatic glucose production, in SDT rats was not different from that in SD rats. GK has been thought to be essential for liver in maintaining metabolic function because it exerts a strong influence on glucose utilization by stimulating glycolysis and glycogen synthesis in liver [19]. GK activity decreased in the liver of alloxan-diabetic rats and the activity was improved by beta 3 -adrenagic agonists [20]. Also, peroxisome proliferator-activated receptor (PPAR) $-\gamma$ agonists enhanced GK activity and expression 


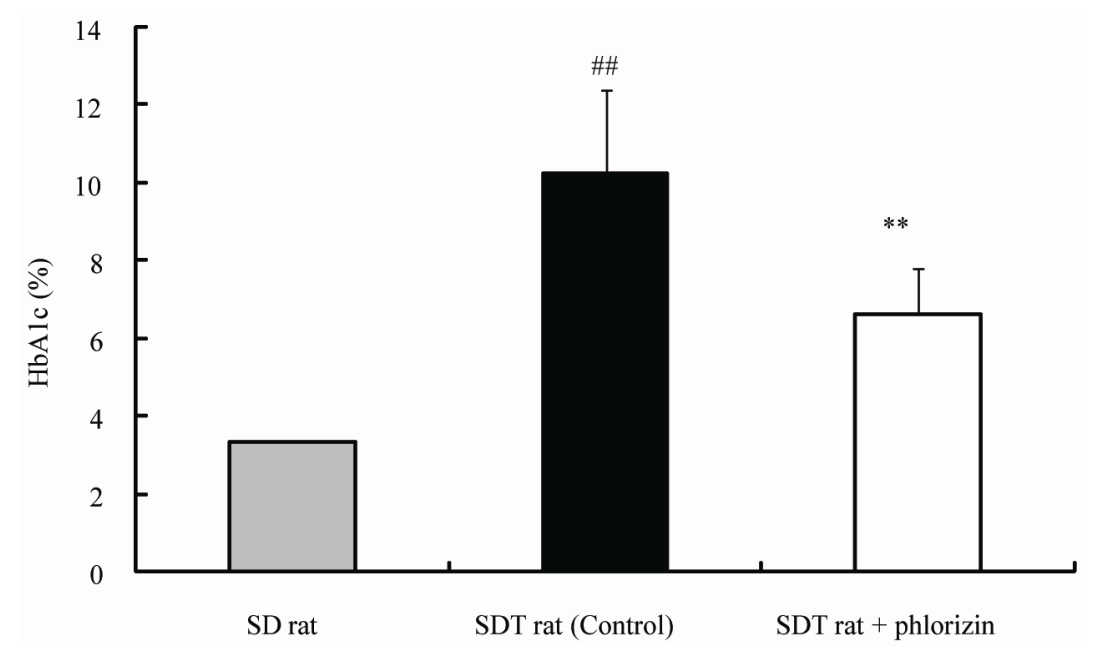

Figure 2. Effect of phlorizin on HbA1c level in SDT rats. Data represent mean \pm standard deviation $(\mathrm{n}=5-6)$. ${ }^{\#} \mathrm{p}<0.01$; significantly different from SD rat. ${ }^{* *} \mathrm{p}<$ 0.01 ; significantly different from SDT rat (Control).

Table 2. mRNA expression of enzymes and proteins related to glycolipid metabolism in liver (copy/106 copy $18 \mathrm{~s}$ rRNA).

\begin{tabular}{lccc}
\hline Enzyme/protein & SD rat & SDT rat (Control) & SDT rat + Phlorizin \\
\hline GK & $2.94 \pm 0.86$ & $0.07 \pm 0.05^{\# \#}$ & $0.90 \pm 0.76^{*}$ \\
G-6-Pase & $13.44 \pm 11.59$ & $13.55 \pm 5.71$ & $27.49 \pm 26.33$ \\
GS & $0.71 \pm 0.33$ & $0.40 \pm 0.08$ & $1.04 \pm 0.60^{*}$ \\
GP & $2.40 \pm 0.94$ & $0.76 \pm 0.19^{\# \#}$ & $2.00 \pm 0.85^{*}$ \\
SREBP1 & $4.07 \pm 1.98$ & $0.43 \pm 0.28^{\#}$ & $1.50 \pm 0.73^{*}$ \\
MTP & $1.23 \pm 0.55$ & $0.43 \pm 0.09^{\#}$ & $1.13 \pm 0.45^{*}$ \\
\hline
\end{tabular}

Data represents mean \pm standard deviation $(\mathrm{n}=5-6) .{ }^{\#} \mathrm{p}<0.05$, ${ }^{\#} \mathrm{p}<0.01$; significantly different from SD rat. ${ }^{*} \mathrm{p}<0.05$; significantly different from SDT rat (Control).

\section{$[19,21]$.}

mRNA expression of GP, which is a key enzyme in glycogen degradation, in SDT rats decreased as compared with that in SD rats (Table 2). The mRNA expression in SDT rats showed a significant increase by phlorizin treatment. mRNA expression of GS in SDT rats showed a tendency of decrease $(p=0.08)$ as compared with that in SD rats. Also, the mRNA expression in SDT rats is increased significantly by phlorizin treatment. GP and GS are the two key regulatory enzymes that catalyze the rate-limiting steps of glycogen degradation and synthesis, respectively. The liver plays a central role in the maintenance of blood glucose homeostasis. Glycogen metabolism in hepatic tissue is one of the major metabolic processes involved in such homeostasis [22]. STZ rats showed a decrease in the mRNA expression of GP but not of GS. Moreover, the reduction in GP was normalized by insulin administration [22]. The changes in GP- and GS-mRNA expression in SDT rats were similar to those in STZ rats.
mRNA expressions associated with lipid metabolism, such as SREBP1 and MTP, showed low expression in SDT rats as compared with SD rats. In phlorizin administration, the MTP and the SREBP1 mRNA expressions increased in SDT rats (Table 2). It is reported that SREBP1 is induced by insulin in liver [23,24]. The decrease in SREBP1 mRNA expression in SDT rats is considered to be related to the decrease in blood insulin levels (Figure 1(c)). On the other hand, it is reported that hepatic MTP mRNA expression is negatively regulated by insulin [25]. MTP mRNA expression in liver of STZ rats significantly increased [26]. It is also reported that hepatic MTP mRNA expression increased in obese models [27]. The hepatic MTP mRNA expression in SDT rats was different from that in other animal models as previously reported. The decrease of lipid-associated mRNA expression might induce a decrease in serum TC levels in SDT rats (Figure 1(e)). It is essential to elucidate the lipid metabolic profile of SDT rats in further studies.

In conclusion, chronic administration of phlorizin in SDT rats produced good glycemic control and an improvement in liver function. Moreover, SDT rats showed a remarkable hyperglycemia, but the lipid levels were not high. The SDT rat is a useful model for pathophysiological investigation of glucotoxicity.

\section{REFERENCES}

[1] Buchanan, T.A. (2003) Pancreatic beta-cell loss and preservation in type 2 diabetes. Clinical Therapeutics, 25, B32-B46. doi:10.1016/S0149-2918(03)80241-2

[2] Kahn, S.E., Prigeon, R.L., Schwartz, R.S., Fujimoto, W.Y., Knopp, R.H., Brunzell, J.D. and Porte, D. Jr. (2001) Obesity, body fat distribution, insulin sensitivity and islet beta-cell function as explanation for metabolic diversity. 
Journal of Nutrition, 131, 354S-360S.

[3] Kanh, S.E. (2003) The relative contribution of insulin resistance and beta-cell dysfunction to the pathophysiology of type 2 diabetes. Diabetologia, 46, 3-19.

[4] LeRoith, D. (2002) Beta-cell dysfunction and insulin resistance in type 2 diabetes: Role of metabolic and genetic abnormalities. American Journal of Medicine, 113, 3S11S. doi:10.1016/S0002-9343(02)01276-7

[5] Robertson, R.P., Harmon, J., Tran, P.O., Tanaka, Y. and Takahashi, H. (2003) Glucose toxicity in $\beta$-cells: Type 2 diabetes, good radical gone bad, and the glutathione connection. Diabetes, 52, 581-587.

doi:10.2337/diabetes.52.3.581

[6] Masuyama, T., Komeda, K., Hara, A., Noda, M., Shinohara, M., Oikawa, T., Kanazawa, Y. and Taniguchi, K. (2004) Chronological characterization of diabetes development in male Spontaneously Diabetic Torii rats. Biochemical and Biophysical Research Communications, 314, 870-877. doi:10.1016/j.bbrc.2003.12.180

[7] Shinohara, M., Masuyama, T., Shoda, T., Takahashi, T., Katsuda, Y., Komeda, K., Kuroki, M., Kakehashi, A. and Kanazawa, Y. (2000) A new spontaneously diabetic nonobese Torii rat strain with severe ocular complications. International Journal of Experimental Diabetes Research, 1, 89-100. doi:10.1155/EDR.2000.89

[8] Ohta, T., Matsui, K., Miyajima, K., Sasase, T., Masuyama, T., Shoda, T., Koizumi, K., Shinohara, M. and Matsushita, M. (2007) Effect of insulin therapy on renal changes in Spontaneously Diabetic Torii rats. Experimental Animals, 56, 355-362. doi: 10.1538/expanim.56.355

[9] Sasase, T., Ohta, T., Ogawa, N., Miyajima, K., Ito, M., Yamamoto, H., Morinaga, H. and Matsushita, M. (2006) Preventive effects of glycaemic control on ocular complications of Spontaneously Diabetic Torii rats. Diabetes Obesity and Metabolism, 8, 501-507.

[10] Matsui, K., Oda, T., Nishizawa, E., Sano, R., Yamamoto, H., Fukuda, S., Sasase, T., Miyajima, K., Ueda, N., Ishii Y., Ohta, T. and Matsushita, M. (2009) Pancreatic function of Spontaneously Diabetic Torii rats in pre-diabetic stage. Experimental Animals, 58, 363-374. doi:10.1538/expanim.58.363

[11] Ohta, T., Miyajima, K. and Yamada, T. (2010) Changes in glycolipid metabolism during a high-sucrose feeding in Spontaneously Diabetic Torii (SDT) rats, a genetic model of nonobese type 2 diabetes. Journal of Animal and Veterinary Advances, 9, 2883-2889. doi:10.3923/javaa.2010.2883.2889

[12] Ohta, T., Miyajima, K. and Yamada, T. (2011) Pathophysiological changes in pre-diabetic stage of Spontaneously Diabetic Torii (SDT) rats. Journal of Animal and Veterinary Advances, 10, 813-817. doi:10.3923/javaa.2011.813.817

[13] Boccia, M.M., Kopf, S.R. and Baratti, C.M. (1999) Phlorizin, a competitive inhibitor of glucose transport, facilitates memory storage in mice. Neurobiology of Learning and Memory, 71, 104-112. doi:10.1006/nlme.1998.3856

[14] Crespy, V., Aprikian, O., Morand, C., Besson, C., Manach, C., Demigne, C. and Reitman, C. (2001) Bioavailability of phloretin and phloridzin in rats. Journal of Nutrition, 131, 3227-3230.

[15] Burcelin, R., Mrejen, C., Decaux, J.F., De Mouzon, S.H., Girard, J. and Charron, M.J. (1998) In vivo and in vitro regulation of hepatic glucagon receptor mRNA concentration by glucose metabolism. The Journal of Biological Chemistry, 273, 8088-8093. doi:10.1074/jbc.273.14.8088

[16] Rossetti, L., Smith, D., Shulman, G.I., Papachristou, D. and DeFronzo, R.A. (1987) Correction of hyperglycemia with phlorizin normalizes tissue sensitivity to insulin in diabetic rats. The Journal of Clinical Investigation, 79, 1510-1515. doi:10.1172/JCI112981

[17] Ling, Z.C., Hong-Lie, C., Östenson, C.-G., Efendic, S. and Khan, A. (2001) Hyperglycemia contributes to impaired insulin response in GK rat islets. Diabetes, 50, S108-S112. doi:10.2337/diabetes.50.2007.S108

[18] McCrimmon, R.J., Evans, M.L., Jacob, R.J., Fan, X., Zhu, Y., Shulman, G.I. and Sherwin, R.S. (2002) AICAR and phlorizin reverse the hypoglycemia-specific defect in glucagon secretion in the diabetic BB rat. American Journal of Physiology-Endocrinology and Metabolism, 283, E1076-E1083.

[19] Ferre, T., Riu, E., Bosch, F. and Valera, A. (1996) Evidence from transgenic mice that glucokinase is rate limiting for glucose utilization in the liver. The FASEB Journal, 10, 1213-1218.

[20] Milagro, F.I., Gómez-Ambrosi, J., Forga, L. and Martinéz, J.A. (1999) A beta3-adrenergic agonist increases muscle GLUT1/GLUT4 ratio, and regulates liver glucose utilization in diabetic rats. Diabetes Obesity and Metabolism, 1, 97-104. doi:10.1046/j.1463-1326.1999.00019.x

[21] Kim, S.-Y., Kim, H.-I., Park, S.-K., Im, S.-S., Li, T., Cheon, H.G. and Ahn, Y.-H. (2004) Liver glucokinase can be activated by peroxisome proliferator-activated receptor- $\gamma$. Diabetes, 53, S66-S70. doi:10.2337/diabetes.53.2007.S66

[22] Rao, P.V., Pugazhenthi, S. and Khandelwal, R.L. (1995) The effects of streptozotocin-induced diabetes and insulin supplementation on expression of the glycogen phosphorylase gene in rat liver. The Journal of Biological Chemistry, 270, 24955-24960. doi:10.1074/jbc.270.42.24955

[23] Ribaux, P.G. and Iynedjian, P.B. (2003) Analysis of the role of protein kinase B (cAKT) in insulin-dependent induction of glucokinase and sterol regulatory elementbinding protein 1 (SREBP1) mRNAs in hepatocytes. Biochemical Journal, 376, 697-705. doi:10.1042/BJ20031287

[24] Foretz, M., Pacot, C., Dugail, I., Lemarchand, P., Guichard, C., Le, liepvre, X., Berthelier-Lubrano, C., Spiegelman, B., Kim, J.B. and Ferre, P. (1999) ADD1/SREBP1c is required in the activation of hepatic lipogenic gene expression by glucose. Molucular and Cellular Biology, 19, 3760-3768.

[25] Sparks, J.D., Chamberlain, J.M., O’Dell, C., Khatun, I. and Hussain, M.M. (2011) Acute suppression of apo B secretion by insulin occurs independently of MTP. Biochemical and Biophysical Research Communications, 
406, 252-256. doi:10.1016/i.bbrc.2011.02.028

[26] Lally. S., Owens, D. and Tomkin, G.H. (2007) Genes that affect cholesterol synthesis, cholesterol absorption, and chylomicron assembly: The relationship between the liver and intestine in control and streptozotosin diabetic rats. Metabolism Clinical and Experimental, 56, 430-438. doi:10.1016/j.metabol.2006.10.028

[27] Phillips, C., Owens, D., Collins, P. and Tomkin, G.H. (2002) Microsomal triglyceride transfer protein: Does insulin resistance play a role in the regulation of chylomicron assembly? Atherosclerosis, 160, 355-360. doi:10.1016/S0021-9150(01)00721-3 